\title{
SPATIAL NUMERICAL RANGES OF ELEMENTS OF BANACH ALGEBRAS
}

\author{
A.K. GAUR \\ Department of Mathematics \\ Duquesne University \\ Pittsburgh, PA 15282, IJ.S.A. \\ and

\section{T. HUSAIN} \\ Department of Mathematics and Statistics \\ McMaster University \\ Hamilton, Ontario \\ L8S 4K1 Canada
}

(Received August 30, 1988 and in revised form January 19, 1989)

\begin{abstract}
In this paper, the notion of spatial numerical range of elements of Banach algebras without identity is studied. Specifically, the relationship between spatial numerical ranges, numerical ranges and spectra is investigated. Among other results, it is shown that the closure of the spatial numerical range of an element of a Banach algebra without identity but with regular norm is exactly its numerical range as an element of the unitized algebra. Futhermore, the closure of the spatial numerical range of a hermitian element coincides with the convex hull of its spectrum. In particular, spatial numerical ranges of the elements of the Banach algebra $C_{0}(X)$ are described.
\end{abstract}

KEY WORDS AND PHRASES. Spatial numerical ranges, numerical ranges, spectrum, regular norm of a normed or Banach algebra.

1980 AMS SUBJECT CLASSIFICATION CODES. 46J99, 46J15.

1. INTRODUCTION.

Let $A$ be a Banach algebra with identity $1,\|1\|=1$. For each $x \in A$, the subset of the complex numbers,

$$
V(A, x)=\left\{f(x): f \in A^{\prime}, f(1)=1=\|f\|\right\}
$$

is called the numerical range of $x$, where $A^{\prime}$ is the dual of $A$, (see [1], [2]). Evidently, this definition is dependent on the identity of the Banach algebra. Since a Banach algebra need not have an identity, it is worthwhile to study this notion for elements of Banach algebras without identity. The purpose of this paper is to investigate this situation. 
Bonsall and Duncan [1] have considered the following situation: Let $B(A)$ denote the Banach algebra of all bounded linear operators on $A$. For each a $\varepsilon A$, put $T_{a}(x)=$ $\operatorname{ax}(x \in A)$, then $T_{a} \in B(A)$ and $I$ (identity operator) is the identity of $B(A)$. They show [1] that

$$
V\left(B(A), T_{a}\right)=U_{x \in S(A)}\left\{f(a x): E \in A^{\prime},\|f\|=1=f(x)\right\}
$$

where $S(A)=\{x \in A:\|x\|=1\}$ and call it the spatial numerical range of a $\varepsilon$. We wish to adopt this notion (Definition 2.1.) to define spatial numerical range for an element of an arbitrary Banach algebra without identity. For this, we require a condition on the norm of the Banach algebra. Specifically, we say that the norm of a normed algebra $A$ is regular (see [3] for details) if for each $x \varepsilon A$,

$$
\|\mid x\|=\sup \{\|a x\|:\|a\|<1 \text {, a } \varepsilon A\} \text {. }
$$

Each Banach algebra with an identity can be given an equivalent regular norm. We wil1 show (Remark 3.8) why the regularity of the norm in this connection is relevant.

Let $A$ be a Banach algebra without identity but with regular norm. Among the results, we prove that the convex closure of the spatial numerical range $V_{A}(x)$ (Def. 2.1) of $x \in A$ coincides with the numerical range of $x \in A^{+}$1.e. $\overline{c o}^{-} V_{A}(x)=V\left(A^{+}, x\right)$ (Theorem 2.3). Moreover, the spatial numerical range $V_{A}(h)$ of a hermitian element $h \varepsilon A$ is connected and $\bar{v}_{A} \overline{(h)}=c o \operatorname{sp}_{A}(h)$ (convex hull of the spectrum of $h$ )(Theorem 3.5). We apply these results to describe spatial numerical ranges of elements of the commutative Banach algebra $C_{0}(X)$ of all continuous complex-valued functions vanishing at infinity on a locally compact, noncompact, Hausdorff space X (Theorem 4.1). We show that the spatial numerical range of an element in $C_{0}(X)$ lies in between the convex hull and convex closure of its range in the complex plane $\mathbb{C}$.

All Banach algebras in the sequel are over the complex field $C$ unless otherwise stated.

2. SPATIAL NUMERICAL RANGES OF ARBITRARY ELEMENTS.

Let $A$ denote a Banach algebra. For each $x \in A$, let

$$
D_{A}(x)=\left\{f \varepsilon A^{\prime}:|| f||=1=f(x)\right\} \text {. }
$$

By a corollary of the Hahn-Banach theorem, $D_{A}(x)$ is nonempty. As before, we set $S(A)=\{x \in A:|| x||=1\}$. We have

DEFINITION 2.1

(1) For each a $\varepsilon A$, we call

$$
V_{A}(a)=\left\{f(a x): f \in D_{A}(x), x \in S(A)\right\}
$$

the spatial numerical range of a.

(11) The nonnegative real number

$$
\nu_{A}(a)=\sup \left\{|\lambda|: \lambda \varepsilon V_{A}(a)\right\}
$$

will be called the spatial numerical radius of $a$.

We recall [3] that the relative numerical range of a $\varepsilon A$ with respect to $x$, $x \in S(A)$ is

$$
V_{X}(A, a)=\left\{f(a x): F \in D_{A}(x)\right\}
$$


Thus we see that $V_{A}(a)=U\left\{V_{x}(A, a): x \in S(A)\right\}$ which is a bounded subset of the complex numbers, bounded by $\|a\|$. Furthermore, for any a $\varepsilon A, T a \varepsilon B$ (A) (defined in the introduction) we have:

$$
V_{A}(a)=V\left(B(A), T_{a}\right) \text {. }
$$

This justifies the usage of the notion of spatial numerical range as defined by Bonsall and Duncan [1].

The following statements are immediate consequences of the definitions concerned: LEMMA 2.2 Let $\mathrm{a}, \mathrm{b} \in \mathrm{A}$ and $\alpha \in \mathbb{C}$. Then

$$
\begin{aligned}
& v_{A}(a+b) \quad v_{A}(a)+v_{A}(b) \\
& v_{A}(\alpha a)=\alpha v_{A}(a) \\
& v_{A}(a+b)<v_{A}(a)+v_{A}(b)
\end{aligned}
$$

THEOREM 2.3 Let A be a Banach algebra without identity. If A has regular norm, then for each a $\varepsilon A$,

$$
\overline{c o} V_{A}(a)=V\left(A^{+}, a\right) \text {. }
$$

(co $\mathrm{X}$ denotes the closure of the convex hull of $X_{.}$)

PROOF. For each $x \in A, \lambda \varepsilon \mathbb{C}$, define

$$
T_{(\lambda, x)}(a)=\lambda a+x a \quad(a \notin A) .
$$

Then $T_{(\lambda, x)} \varepsilon B(A)$. We set $\Psi: A^{+} \rightarrow B(A)$ by

$$
\Psi(\lambda, \mathbf{x})=T_{(\lambda, x)}
$$

Clearly, $\Psi$ is an algebra homomorphism. If $\Psi(\lambda, x)=0$, then for all a $\varepsilon A$, $\lambda a+x a=0$ and so $a=\left[-\frac{x}{\lambda}\right]$ a if $\lambda \neq 0$. Thus $-\frac{x}{\lambda}$ for $\lambda \neq 0$, is an identity of $A$ which is ruled out by hypothesis. If $\lambda=0$, then $x a=0$. By the regularity of norm, $\|x\|=|| a\left|\|_{<1}^{\text {sup }}\right||x a| \mid=0$, showing that $(\lambda, x)=(0,0)$. Thus $\Psi$ is injective. We endow $A^{+}$with another equivalent norm ||$(\lambda, x)\left\|_{+}=\right\| T(\lambda, x) \|$. But then for each $\mathbf{x} \varepsilon \mathbf{A}$,

$$
\|x\|_{+}=\|(0, x)\|_{+}=\left\|x_{(0, x)}\right\|=\sup _{\| a||<1}\|x a\|=\|x\|,
$$

because the norm of $A$ is regular. This proves that $\Psi$ is an isometry from $A$ into $B(A)$ such that $\Psi(1,0)=I$. Now by result $([1]$, p.84), the theorem follows.

COROLLARY 2.4 Let $A$ be the same as in Theorem 2.3. Then $\nu_{A}$ (Def. 2.1.) defines an equivalent norm on $A$ and for each $x \in A$,

where $e=\exp (1)$.

$$
e^{-1}\|x\|<v_{A}(x)<\|x\|,
$$


PROOF. First we show that $\nu_{A}(x)=\nu_{A}(x)$ for each $x \in A$. For this, it is sufficient to show that the following two sets $D_{1}, D_{2}$ are equal, where $D_{1}=\{r>0$ : $\left.V\left(A^{+}, x\right) \subset D(r)\right\}$ and $D_{2}=\left\{r>0: V_{A}(x) \subset D(r)\right\}$ in which $D(r)$ is the closed disk of radius $r$ centered at 0 in $C$. If $r \varepsilon D_{1}$ then $D(r) \supset V\left(A^{+}, x\right) \supset V_{A}(x)$ by Theorem 3.2. and so $r \in D_{1}$. On the other hand, if $s \in D_{2}$ then $D(s) \supset V_{A}(x)$ implies $D(s) \supset \overline{c o} v_{A}(x)$

$V\left(A^{+}, x\right)$ because $D(s)$ is closed and convex. Thus $s \varepsilon D_{1}$ and so $D_{1}=D_{2}$. But then for all $x \in A$

$$
\nu_{A}(x)=\inf D_{1}=\text { inf } D_{2}=v_{A^{+}}(x)
$$

Since $\mathrm{A}^{+}$is a unital Banach algebra, by the result ([1], p.34) we conclude

$$
\|x\|=\|(0, x)\|_{+}>v_{A^{+}}(x)>e^{-1}||(0, x)\left\|_{+}=e^{-1}\right\| x \|_{+} \cdot
$$

COROLLARY 2.5 For A as in Theorem 2.3., the following statements are equivalent:

(i) For each a $\varepsilon A, \overline{c o} \operatorname{sp}_{A}(a)=\overline{c o} v_{A}(a)$.

(ii) For each a $\varepsilon A$, co $\left\{\lambda^{2}: \lambda \varepsilon V\left(A^{+}, a\right)\right\} \supset V\left(A^{+}, a^{2}\right)$.

PROOF. Since $\mathrm{sp}_{\mathrm{A}}(\mathrm{a})=\mathrm{sp}_{\mathrm{A}}(\mathrm{a})[5]$, in view of Theorem 2.3, we have

$$
\left.\overline{c o} \operatorname{sp}_{A}(a)=\overline{c o} \operatorname{sp}_{A^{+}}(a)=v_{\left(A^{+}\right.}, a\right)=\overline{c o} v_{A}(x)
$$

by a Bonsall and Duncan's result ([2], p.49). Now the corollary follows from [2].

\section{SPATIAL NUMERICAL RANGES OF HERMITIAN ELEMENTS}

In this section, A continues to stand for a Banach algebra without identity. Here we study the spatial numerical range of hermitian and positive elements.

DEFINITION 3.1 An element a $\varepsilon A$ is called hermitian if $V_{A}(a) \subset I R$ (the set of real numbers). If $\mathrm{V}_{\mathrm{A}}(\mathrm{a}) \subset \mathbb{R}^{+}$, a is called a positive element. Let $\mathrm{H}(\mathrm{A})$ (resp. $\mathrm{K}(\mathrm{A})$ ) denote the set of all hermitian (resp. positive) elements.

PROPOSITION 3.2 For any Banch algebra $A$ with regular norm,

$$
H(A)=A \cap H\left(A^{+}\right) \text {. }
$$

PROOF. By Theorem 2.3., $V_{A}(x) \subset \mathbb{R}$ iff $V\left(A^{+}, x\right) \subset \mathbb{R}$ and the proposition follows .

COROLLARY 3.3 Let $A$ be a $B^{*}$-algebra with regular norm. Then $h \in H(A)$ iff $h=h^{*}$. PROOF. Since $A$ has regular norm, by Proposition 3.2., $H(A)=A \cap H\left(A^{+}\right)$and so by the result ( $[1], \mathrm{p} .47) \mathrm{h} \varepsilon \mathrm{H}\left(\mathrm{A}^{+}\right)$iff $h=h^{*}$.

COROLLARY 3.4 For $A$ as in Proposition 3.2., $H(A)$ is a real Banach space and for each $h \varepsilon H(A)$,

$$
\mathrm{v}_{A}(h) \subset \cos \operatorname{sp}_{A}(h) \text {. }
$$

PROOF. It is easy to verify that $H(A)$ is a real Banach space. By Proposition 3.2. [3] and the result ([1], p.53), $v_{A}(h) \subset \cos \operatorname{sp}_{A+}(h)=\cos \operatorname{sp}_{A}(h)$ ([5).

THEOREM 3.5 Let A be a Banach algebra with regular norm. Then for each $h \in H(A)$,

$$
\overline{V_{A}(h)}=\operatorname{coss} A_{A}(h) .
$$


PROOF. As pointed out above after Definition 2.l., $V_{A}(h)=V\left(B(A), T_{h}\right)$ which is connected by a result in [6] and hence convex. Since $V_{A}(h) \subset \mathbb{R}$, by Theorem 2.3, we conclude

$$
\overline{\operatorname{co}} v_{A}(h)=V\left(A^{+}, h\right)=\overline{v_{A}(h)}
$$

But then from ( $[1]$, p.53) it follows that

by $[5]$.

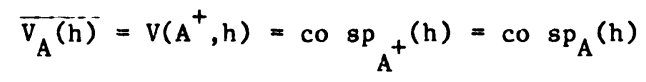

We give a more explicit description of Proposition 3.2.

THEOREM 3.6. Let $A$ be a Banach algebra without unit but with regular norm. Then $(\lambda, h) \in H\left(A^{+}\right)$iff $h \varepsilon H(A)$ and $\lambda \varepsilon \mathbb{R}$.

PROOF. If $(\lambda, h) \in H\left(A^{+}\right)$, then $V\left(A^{+},(\lambda, h)\right) \subset \mathbb{R}$ and so far for all $f \in\left(A^{+}\right)^{\prime},\|f\|$ $=1=f\left((1,0), f\left((\lambda, h) \varepsilon \mathbb{R}\right.\right.$. Since $A$ has regular norm, the mapping $a+T_{a}$ of $A$ into $B(A)$ is an isometry (Theorem 2.3) and so $T_{A}=\left\{T_{a}: a \in A\right\}$ is a closed subset of $B(A)$ and $\mathrm{I} k \mathrm{~T}_{\mathrm{A}}$. Let

$$
\operatorname{dis}\left(I, T_{A}\right)=\operatorname{Inf}\left\{\left\|I-T_{a}\right\|: a \varepsilon A\right\}
$$

Since $0 \in A$ implies $T_{0}=0$, we have $\operatorname{dis}\left(I, T_{A}\right)<1$. To show $\operatorname{dis}\left(I, T_{A}\right)=1$, assume it is < 1. Then there is a $\varepsilon A$ with $\left\|I-T_{a}\right\|<1$ and so $T_{a}$ is invertible with $T_{a}^{-1} \varepsilon B(A)$. If $p=T_{a}^{-1} a \varepsilon A$, then for all $x \in A, p x=T_{a}^{-1} a x=x$ shows that $p$ is the identity of $A$, which is ruled out by hypothesis. Thus $\operatorname{dis}\left(I, T_{A}\right)=1$. By a corollary of the Hahn-Banach Theorem [7] there is $f \in\left(A^{+}\right)^{\prime}$ such that $f((1,0))=1$, $f((0, h))=0$. But then $(\lambda, h)=(0, h)+\lambda(1,0)$ implies $f(\lambda, h)=\lambda \varepsilon \mathbb{R}$. Also $(0, h)=(\lambda, h)-\lambda(1,0)$ shows that $h \varepsilon H(A)$ by Corollary 3.4. Clearly, $h \varepsilon H(A)$ and $\lambda \varepsilon \mathbb{R}$ imply that $(\lambda, h) \in H\left(A^{+}\right)$and the proof is complete.

Finally, we have a characterization of positive elements of $A$.

THEOREM 3.7 Let A be a Banach algebra without identity but with regular norm. Then an element $k \in A$ is positive iff $k \in H(A)$ and $\operatorname{sp}_{A}(k) \subset \mathbb{R}^{+}$.

PROOF. Since each positive element is clearly hermitian, by Theorem 2.3., $\operatorname{sp}_{A}(k) \subset V_{A}(k) \subset \mathbb{R}^{+}$. Theorem 3.5. implies the "only if" part.

REMARK 3.8 The justification of considering the regular norm in the above results lies in the following (the authors thank $\mathrm{Z}$. Kovarik for this suggestion). Suppose $\mathrm{A}^{+}$ is endowed with the usual norm $\|(\lambda, x)||=|\lambda|+|| x||$, then the numerical range of $(\lambda, x) \varepsilon A^{+}$is trivial because for $f \varepsilon\left(A^{+}\right)^{\prime}$, we have $f=(\mu, g)\left(\mu \varepsilon C, g \varepsilon A^{\prime}\right)$ with $\| f||=\max (|\mu|, \| f||)$ and $(\mu, g)(1,0)=\mu=1$ which gives $\|(\mu, g)\|=1$ Iff $\| \mathrm{g}||<1$ and so $\{(1, \mathrm{~g})(0, \mathrm{x}):\|\mathrm{g}\|<1\}=\{\mathrm{g}(\mathrm{x}):\|\mathrm{g}\|<1\}=\overline{\mathrm{D}}\|\mathrm{x}\|(0)$, the closed disk of radius $\|x\|$. But this is known in [1].

4. SPATIAL NUMERICAL RANGES OF ELEMENTS OF $\mathrm{C}_{\mathrm{o}}(\mathrm{X})$

In this section, we consider the Banach algebra $C_{0}(X)$ of all continuous complexvalued functions vanishing at infinity on a locally compact Hausdorff space $X$. Indeed 
$C_{0}(X)$ is commutative and has no identity. We wish to describe spatial numerical ranges of elements of $C_{\sigma}(X)$. When $X$ is a compact Hausdorff space, then Stampfli and Williams [4] have shown that the numerical range of each element $f$ of the Banach algebra $C(X)$ of all continuous complex-valued functions on $X$ is the closed convex hull of its range, $f(X)$.

By way of comparision, we also show by an example that the spatial numerical range of an element of a Banach algebra without identity need not be closed whereas it is well known that the numerical range is always closed and convex ([1]).

THEOREM 4.1 Let $X$ be a locally compact Hausdorff space and $C_{0}(X)$ the commutative Banach algebra of all continuous complex-valued functions on $X$ that vanish at infinity. Then for each $g \in C_{0}(X)$,

where $R(g)$ is the range of $g$.

$$
\operatorname{co} R(g) \subset V_{C_{0}(X)}(g) \subset \overline{\operatorname{co}} R(g),
$$

PROOF. Let $\alpha \in$ co $R(g)$. Then $\alpha=\sum_{i=1}^{n} \lambda_{1} g\left(x_{1}\right)$ with $\lambda_{1}>0, \sum_{i=1}^{n} \lambda_{i}=1$ for some $x_{i} \varepsilon X . \quad$ Define

$$
\varphi(h)=\sum_{i=1}^{n} \lambda_{1} h\left(x_{1}\right)
$$

for $h \varepsilon C_{0}(X)$. Clearly $\varphi$ is a bounded linear functional on $\mathrm{C}_{0}(\mathrm{X})$ and $(\mathrm{h})>0$ whenever $h>0$. Thus $\varphi \in C^{\prime}{ }_{0}(X)$ represents a measure with total variation $=\|\varphi\|=1$.

By complete regularity of $X$, there is $f: X+[0,1]$ in $C_{0}(X)$ with $\|f\|=1$ and $f\left(x_{1}\right)=$ $1(1<i<n)$. But then

$$
\begin{aligned}
& \qquad \varphi(f)=\sum_{1=1}^{n} \lambda_{1} f\left(x_{1}\right)=1 \\
& \text { and } \varphi(f g)=\sum_{i=1}^{n} \lambda_{1} f\left(x_{1}\right) g\left(x_{1}\right)=\sum_{i=1}^{n} \lambda_{1} g\left(x_{1}\right)=\alpha . \\
& \text { This proves that co } R(g) \subset v_{C_{0}(x)}(g) .
\end{aligned}
$$

On the other hand, suppose $\alpha \in \mathrm{V}_{C_{0}(X)}(\mathrm{g})$. Then there is $\mathrm{f} \varepsilon \mathrm{C}_{\mathrm{o}}(\mathrm{X}),\|\mathrm{f}\|=1$ with $\alpha \varepsilon V_{f}\left(C_{0}(X), g\right)$ and so there is $\varphi \varepsilon C^{\prime}{ }_{0}(X)$ with $\|\varphi\|=1=\varphi(f)$ such that $\alpha=\varphi(\mathrm{fg})$.

Let $E=\{x \in X:|f(x)|=1\}$. Then $E$ is a compact subset of $X$ because f $\varepsilon C_{0}(X)$. Since $\varphi(f)=1$ and $\|\varphi\|=1, \varphi$ is a measure supported on $E$. Define the function,

$$
w(x)=\left\{\begin{array}{l}
\frac{\overline{f(x)}}{\mid \frac{f(x)}{1}}, x \in E \\
\text { continuously extended to } x \text { with norm less than or equal to } 1 .
\end{array}\right.
$$

Let $M_{w}$ denote the pointwise multiplication by $w$ in $C_{0}(X)$ and let $M^{*}$ be the adjoint of $M$ acting on measures $\psi \varepsilon C_{0}^{\prime}(X)$. If $\vec{w}$ denotes the conjugate of $w$, we define a measure,

$$
\psi=\frac{M^{*} \varphi}{W}
$$


because $w \bar{w}=1$ on $E$ and $M_{w}^{*} \psi=M_{w}^{*} \frac{M^{*}}{w} \varphi=\varphi$.

But then

$$
\begin{aligned}
\alpha & =\varphi(f g)=M_{w}^{*} \psi(f g)=M_{w}^{*} \psi\left(M_{f} g\right) \\
& =\psi\left(M_{w} M_{f} g\right)=\psi\left(M_{w f} g\right) \\
& =\psi(M|f| \varphi)=\psi(|f| g)=\psi(g) .
\end{aligned}
$$

Since $\psi>0,\|\psi\|=1, \psi$ is a probability measure and so

$$
\begin{aligned}
& \alpha=\psi(g)=\lim _{i} \sum \psi\left(E_{i}\right) g\left(x_{i}\right) \quad x_{i} \varepsilon E \\
& \varepsilon \max \psi\left(E_{1}\right) \rightarrow 0 \\
& \operatorname{co} R(g) .
\end{aligned}
$$

This completes the proof.

EXAMPLE 4.2 We show by an example that the spatial numerical range of an element of a Banach algebra without identity need not be closed.

Let $A=\{f \in C[0,1]: f(0)=0\}$. Let $f(x)=x, x \varepsilon[0,1]$. We show that $V_{A}(f)=$ $(0,1]$, which is nonclosed. To see that $0 \notin v_{g}(A, f)$ for $g \in A$ with $\|g\|=1$, suppose the contrary. Then there is $\varphi \varepsilon A^{\prime}$ with $\varphi(\mathrm{g})=1=\|\mathrm{g}\|,\|\varphi\|=1$ such that $\varphi(f g)=0$. Let

$$
E_{g}=\{t \in[0,1]:|g(t)|=1\}
$$

then inf $\mathrm{E}_{\mathrm{g}}>0$. Clearly $\varphi$ is a probability measure with its support in $\mathrm{E}_{\mathrm{g}}$ and thus

$$
0=\varphi(f g)=\int_{E_{g}} f g d \varphi=\int_{E_{g}} \operatorname{tg}(t) d \varphi(t) .
$$

But $g(t) d \varphi(t)$ being a measure with $g(t) d \varphi(t)>0$, we have

$$
0=\varphi(f g)>\operatorname{lnf} E_{g} \int_{E_{g}} g(t) d \varphi(t)>0
$$

(because $\left.t>\operatorname{lnf} \mathrm{E}_{\mathrm{g}}\right)$. This is absurd and $800 k \mathrm{~V}_{\mathrm{g}}(\mathrm{A}, \mathrm{f})$ for any $\mathrm{g} \varepsilon \mathrm{A}$ with $\|\mathrm{g}\|=1$.

Let $t \varepsilon(0,1]$ and construct a continuous function

$$
g_{t}(x)= \begin{cases}0, & \text { if } x=0 \\ 1, & \text { if } x=t \\ \varepsilon[-1,1] & \text { elsewhere }\end{cases}
$$

Let $\varphi=\delta_{t}$ (Dirace measure) so that $\varphi\left(g_{t}\right)=1,\|\varphi\|=1$ and $\varphi(f g)=t$. Thus $(0,1] \subset \mathrm{V}_{\mathrm{A}}(\mathrm{f})$. On the other hand, if $0 \neq \lambda \varepsilon \mathrm{V}_{\mathrm{A}}(\mathrm{f})$, then there $\mathrm{g} \varepsilon \mathrm{A},\|\mathrm{g}\|=1$ with $\lambda=v_{g}(A, f)$. Thus for a $\varphi \varepsilon A^{\prime},\|\varphi\|=1=(g)$, we have

$$
\lambda=\varphi(f g)=\int_{0}^{1} \operatorname{tg}(t) d \varphi(t) \varepsilon(0,1]
$$

because of the properties of the integral and convexity of $(0,1]$. This proves that $V_{A}(t)=(0,1]$, which is not closed. 
ACKNOWLEDGEMENT. This work was partially supported by an NSERC grant.

\section{REFERENCES}

1. BONSALL, F.F. \& DUNCAN, J. Numerical ranges of operators on normed spaces and of elements of normed algebras, London Math. Soc. Lecture Note series 2, 1971 .

2. IBID: Numerical ranges II, Cambridge University Press, 1973.

3. GAUR, A.K. \& HUSAIN, T. Relative numerical ranges, (to appear).

4. STAMPFLI, J. \& WILLIAMS, J.P. Growth conditions and the numerical range in a Banach algebra, Tohoku Math. Jr. 20(1968), 417-424.

5. LARSEN, R. Banach algebras, an Intoduction, Marcel Dekker, Inc. N.Y., 1973.

6. BONSALL, F.F., CAIN, B.E. \& SCHNEIDER, H. The numerical range of a continuous mapping of a normed space, Aequationes Math 2(1968), 86-93.

7. DUNFORD, N. \& SCHWARTZ, J. Linear operators, P.I., Interscience Publishers, Inc. N.Y.. 1958. 


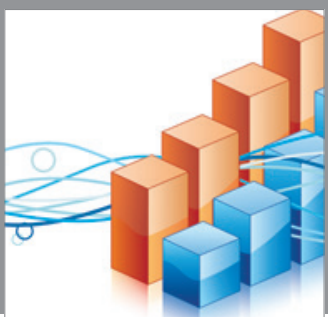

Advances in

Operations Research

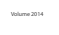

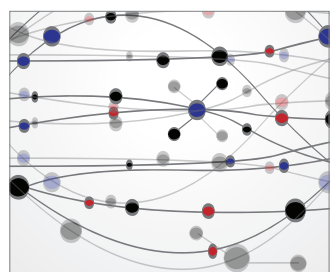

\section{The Scientific} World Journal
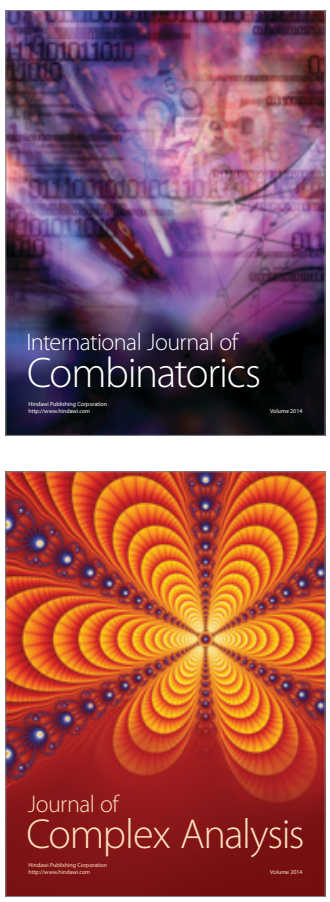

International Journal of

Mathematics and

Mathematical

Sciences
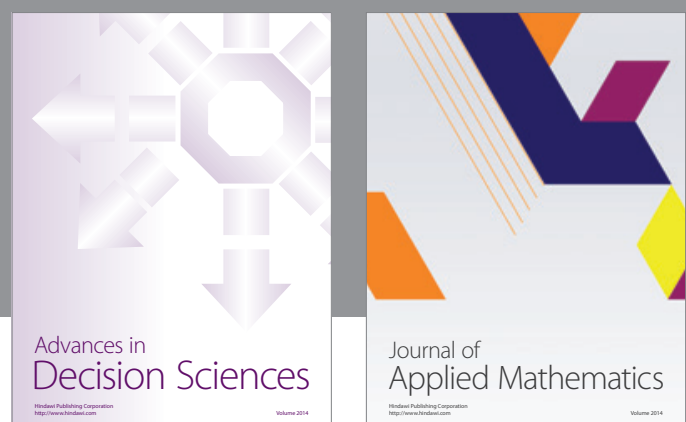

Journal of

Applied Mathematics
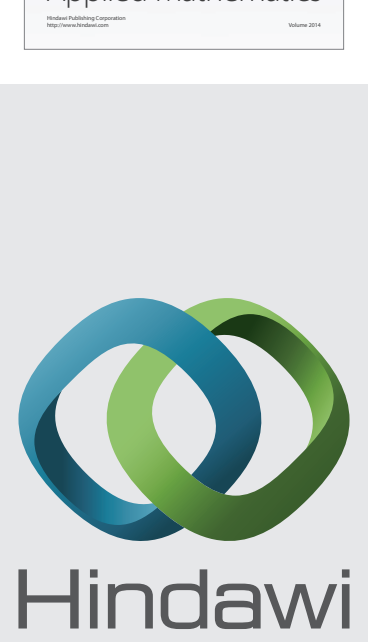

Submit your manuscripts at http://www.hindawi.com
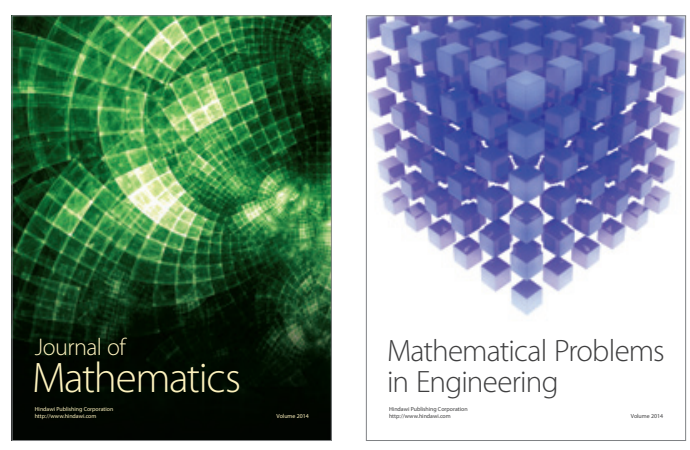

Mathematical Problems in Engineering
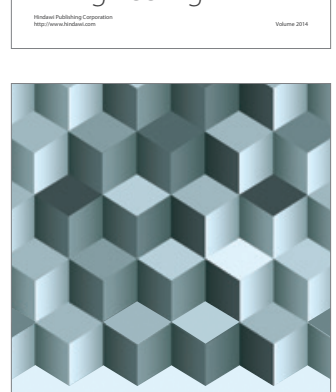

Journal of

Function Spaces
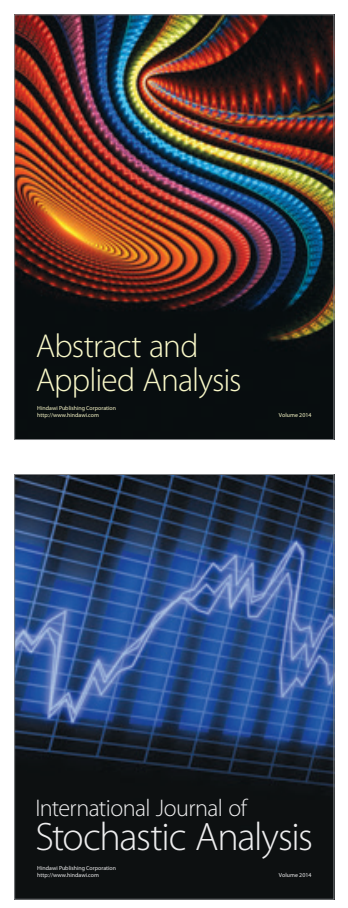

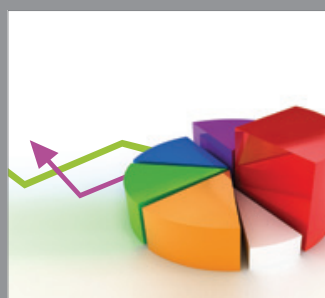

ournal of

Probability and Statistics

Promensencen
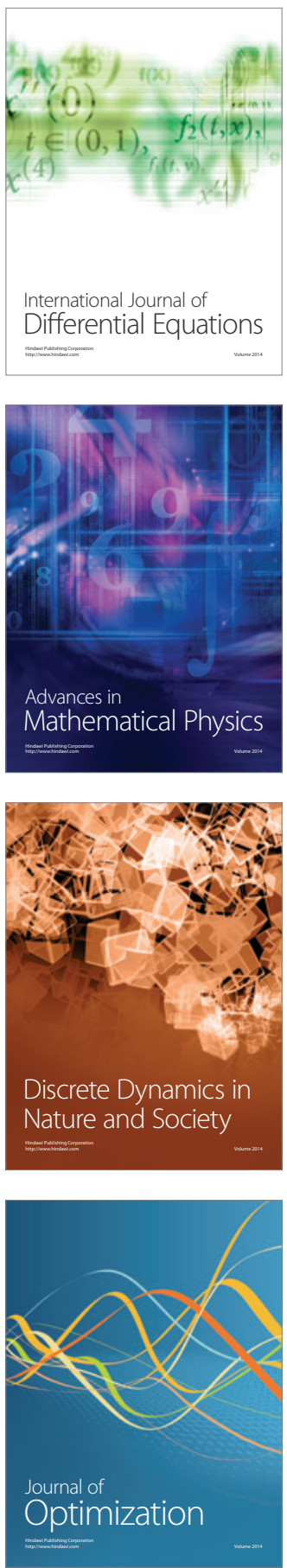